\title{
Simulating the Hip and Knee Behavior of a Biped by Means of Nonlinear Oscillators
}

\author{
Armando Carlos de Pina Filho*
}

Graphical Engineering Department, Universidade Federal do Rio de Janeiro, 21949-900, Rio de Janeiro, RJ, Brazil

\begin{abstract}
Nervous networks in the spinal marrow are capable to produce rhythmic movements, such as: swimming, jumping, and walking. These specialised nervous systems are known as central pattern generators (CPGs). Nonlinear oscillators can be used in control systems of locomotion as pattern generators similar to the pattern of human gait, providing the approach trajectories of the legs. The objective of this work is to present the simulation of the biped gait using a central pattern generator formed by coupled nonlinear oscillators. Using nonlinear oscillators with integer relation of frequency, the transient motion and stable limit cycles of the network formed by oscillators were determined, showing the behavior of the hip and knee angles. Modification of the step length and gait frequency can be obtained by means of the change of few parameters in the oscillators.
\end{abstract}

Keywords: Biped gait, central pattern generator, oscillators.

\section{INTRODUCTION}

The first indications that the spinal marrow could contain the basic nervous system necessary to generate locomotion date back to the early 20th century. Nervous networks in the spinal marrow are capable to produce rhythmic movements, such as: swimming, jumping, and walking, when isolated from the brain and sensorial entrances. These specialised nervous systems are known as central pattern generators (CPGs) [1]. The human locomotion is controlled, in part, by a central pattern generator, which is evidenced in some works [2-4].

Nonlinear oscillators can be used in control systems of locomotion as pattern generators similar to the pattern of human gait, providing the approach trajectories of the legs. The central pattern generator is formed by coupled nonlinear oscillators, where each oscillator generates angular signals of reference for the movement of the legs. Each oscillator has amplitude, frequency and parameters, and coupling terms makes the linking to the other oscillators. Several works on central pattern generators applied in the locomotion of bipeds can be seen in [5-12].

The present work shows a central pattern generator simulated by means of Rayleigh oscillators, which was very little explored in previous research, mainly its application in the locomotion. Despite the apparent similarity with the van der Pol oscillator, the Rayleigh oscillator presents distinct behavior. From electrical point of view, in the van der Pol oscillator, an increase of the voltage implies in increase of the frequency, while in the Rayleigh oscillator this increase of the voltage implies in an increase of the amplitude. This characteristic can make possible a new way of control.

*Address correspondence to this author at the Graphical Engineering Department, Universidade Federal do Rio de Janeiro, 21949-900, Rio de Janeiro/RJ, Brazil. E-mail: pina-filho@deg.ee.ufrj.br
Another important point of the present work is that the proposed system uses mutually coupled oscillators, which represents an advantage in relation to other coupling systems, since the mutual influence between the elements provides a mutual reaction, when one of them is submitted to a disturbance, making possible a most proper functioning of the pattern generator system.

Taking into consideration these aspects, the objective of this work is to present the simulation of the biped gait using a central pattern generator formed by nonlinear oscillators. Some concepts about the human central nervous system, including its main structures and characteristics, are presented. In the analyses, a 2D model with the three most important determinants of gait was considered, that performs only motions parallel to the sagittal plane. Using nonlinear oscillators with integer relation of frequency, the transient motion and stable limit cycles of the network formed by oscillators were determined, showing the behavior of the hip and knee angles. Modification of the step length and gait frequency can be obtained by means of the change of few parameters in the oscillators.

\section{CENTRAL NERVOUS SYSTEM}

The nervous system is constituted by the central and peripheral systems. The peripheral nervous system is constituted by nerves and ganglia. The central nervous system is divided in two main parts: encephalus and spinal marrow. The encephalus is subdivided in cerebrum, cerebellum and cerebral trunk or brainstem. Fig. (1) shows the division of the central nervous system.

The cerebrum is formed by two main parts: diencephalus and telencephalus. Telencephalus is related to the cerebral hemispheres. The function of the diencephalus is to transfer the sensorial information to the cerebral cortex. In relation to the locomotion, it is involved in the regulation of the posture and movement. The motor cortex is part of the cerebral cor- 
tex and its cells supply a direct canal between the cerebrum and the neurons of the spinal marrow, the so-called motoneurons.

The cerebellum is important in the regulation of the fine and complex movements, as well as in the temporal and spatial determination of activation of the muscles in the course of movement or in the postural adjustment [13]. In the locomotion movement, the cerebellum participates actively organising and updating, based in the analysis of sensorial information of position and speed of the limbs at every moment.

The cerebral trunk is formed by three main parts: mesencephalus, pons and bulbus, and summarily, its function is to connect the cerebrum with the spinal marrow, which represents the main way of communication between the cerebrum and the rest of the organism.

The human gait requires a coordination of the muscular activity between the two legs, which is made by a flexible neural coupling to the level of the spinal marrow [14]. Thus, in the course of the locomotion, a disturbance in one of the legs leads to a pattern of proposital reply of the spinal marrow, characterising the existence of the central pattern generator.

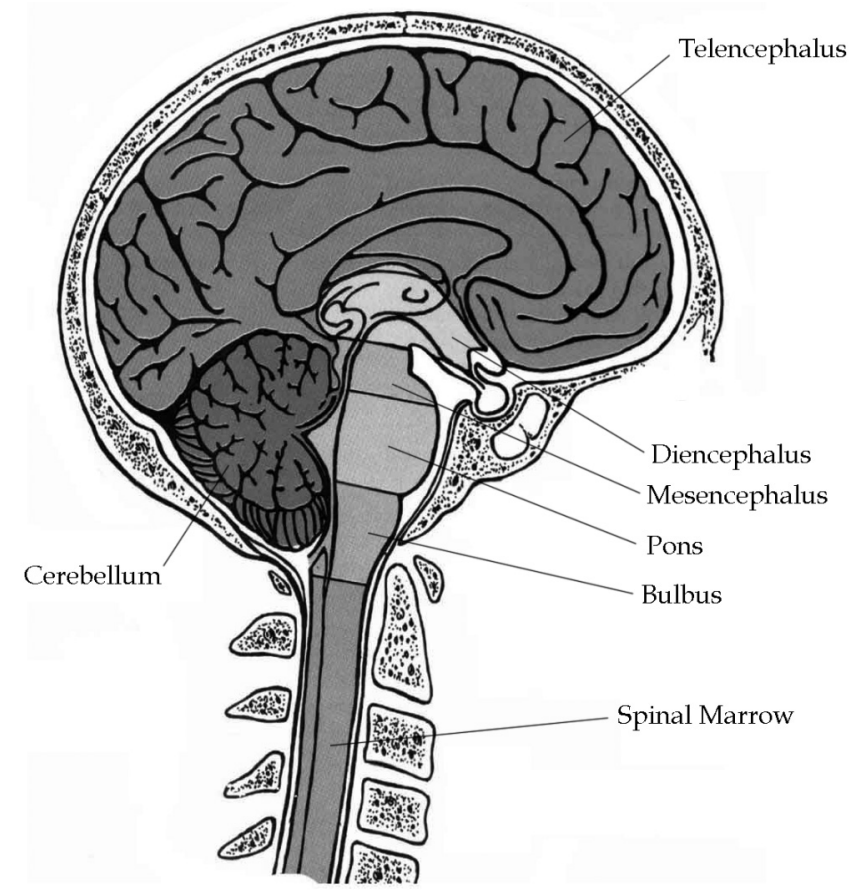

Fig. (1). Division of the central nervous system.

\section{BIPED LOCOMOTION SYSTEM}

Despite people not walking in completely identical way, some characteristics in the gait can be considered universal, and these similar points serve as base for description of patterns of the kinematics, dynamics and muscular activity in the locomotion. The greater interest in this work is related to the patterns of the kinematics, in particular, related to the hip and knee angles. From the knowledge of these patterns of behavior, the simulation of the biped gait using the oscillators system becomes possible.
Fig. (2) presents a scheme of the control system of the human locomotion, controlled by the central nervous system, which the central pattern generator supplies a series of pattern curves for each part of the locomotor. This information is passed to the muscles by means of a network of motoneurons, and the conjoined muscular activity performs the locomotion. Sensorial information about the conditions of the environment or some disturbance are supplied as feedback of the system, providing a fast action proceeding from the central pattern generator, which adapts the gait to the new situation.

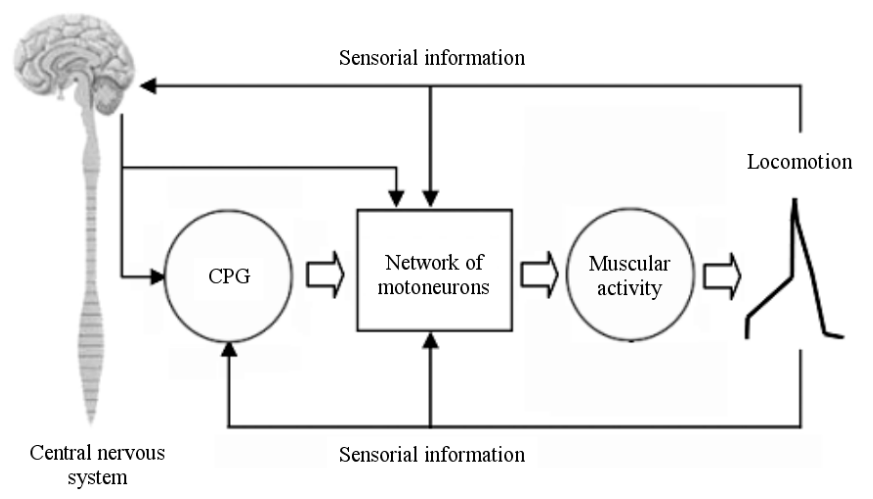

Fig. (2). Control system of the human locomotion.

In relation to the hip, the movements are performed in one only articulation, and these movements can be divided in: flexion or extension, aduction or abduction and external or internal rotation (Fig. 3). This articulation of the hip performs the function of a spherical joint and is formed by the head of femur and acetabulum, located in the pelvis.

In relation to the knee, it represents a articulation with two degrees of freedom, being the flexion-extension the main movement, and the rotation around of the longitudinal axle of the leg is a auxiliary movement, which only appears when the knee is bent. Fig. (4) shows the movements of the knee. More details about the physiology of the articulations of the hip and knee, as well as other parts of the body, can be seen in [15].

Taking into consideration only motions parallel to the sagittal plane, from the use of an optic-electronic system of three-dimensional kinematical analysis [16], it is possible to define the angular behaviour of the hip and knee in the course of the locomotion cycle.

Figs. (5) and (6) present the graphs of angular displacement and phase space of the hip, related to the movements of flexion and extension. Figs. (7) and (8) present the graphs of angular displacement and phase space of the knee, related to the movements of flexion and extension.

\section{BIPED LOCOMOTOR MODEL}

The modeling of the biped gait is made more feasible by reducing the number of degrees of freedom, since there are more than 200 degrees of freedom involved in legged locomotion. The most important determinants of gait are: the compass gait; the pelvic rotation about a vertical axis; the pelvic tilt; the knee flexion of the stance leg; the plantar flex- 
ion of the stance ankle; and the lateral displacement of the pelvis [17].
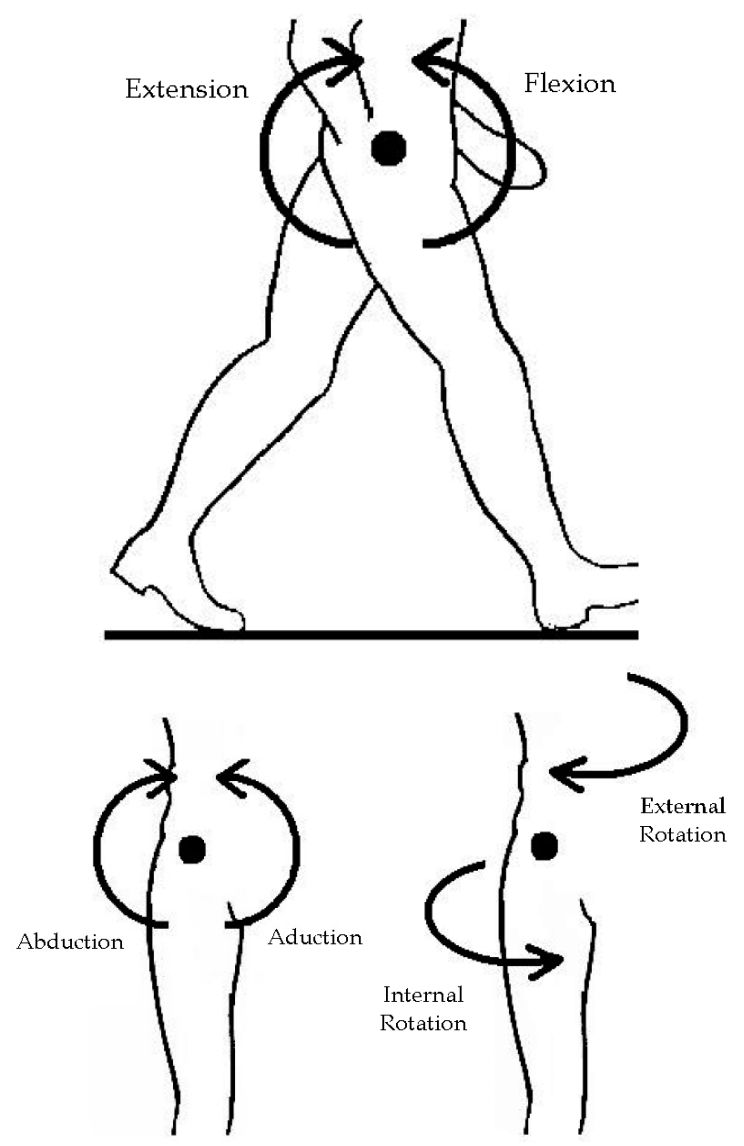

Fig. (3). Movements of the hip.

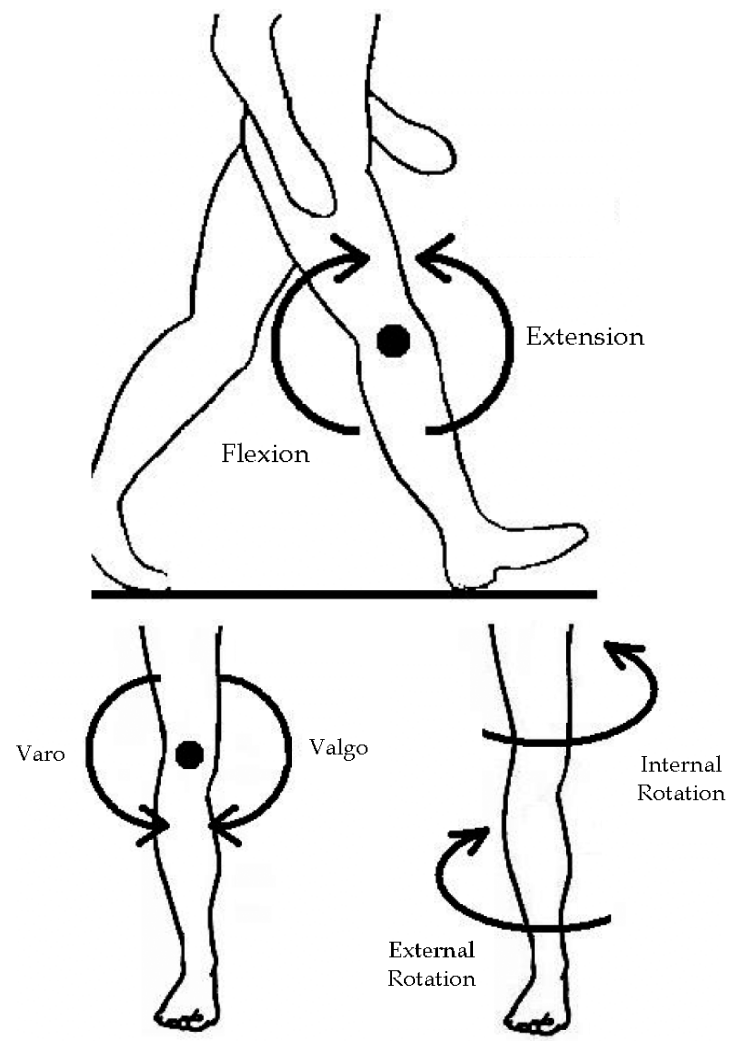

Fig. (4). Movements of the knee.

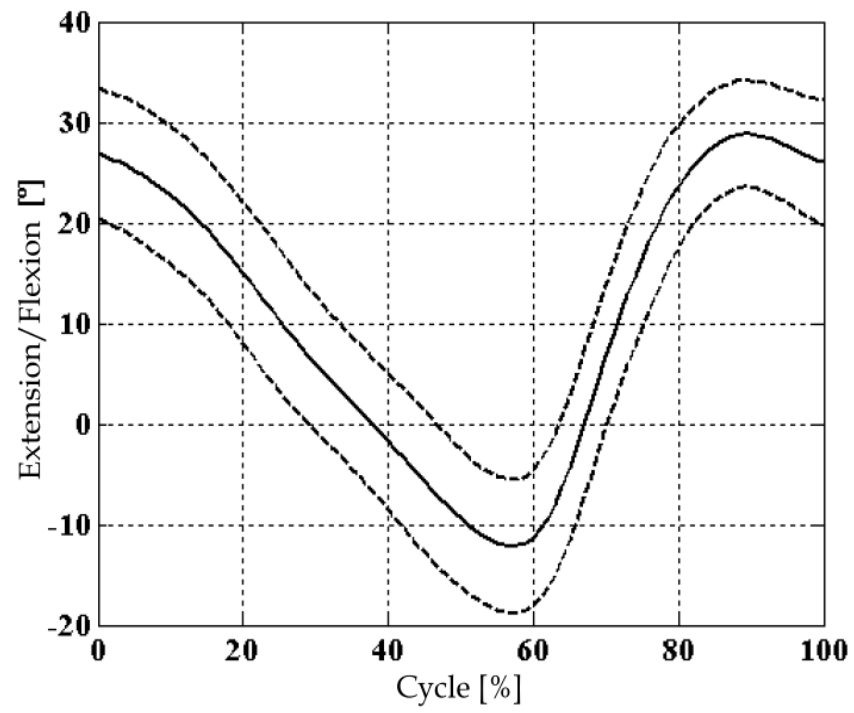

Fig. (5). Angular displacement of the hip in the sagittal plane (mean \pm deviation).

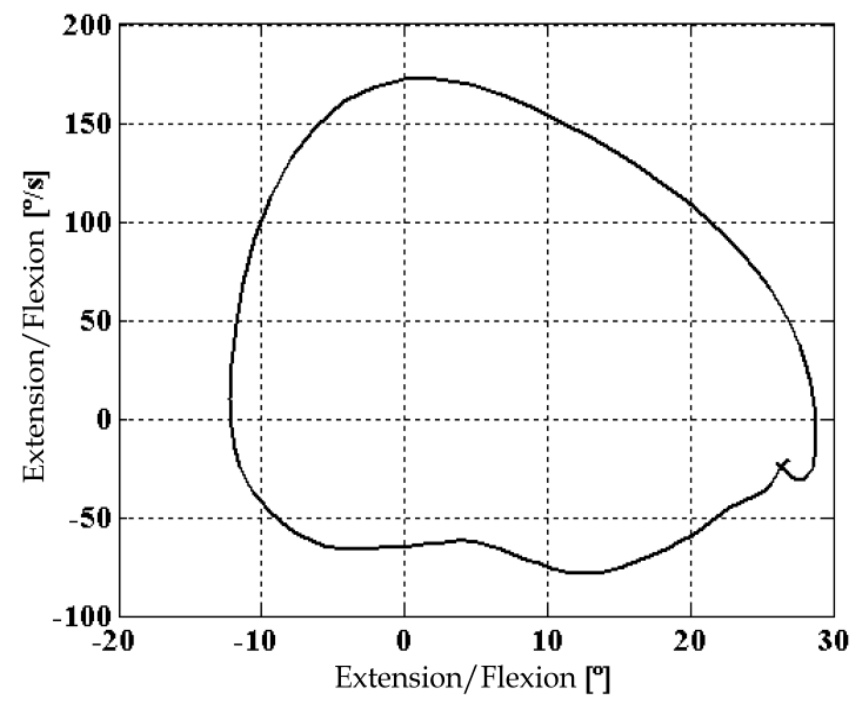

Fig. (6). Phase space of the hip in the sagittal plane.

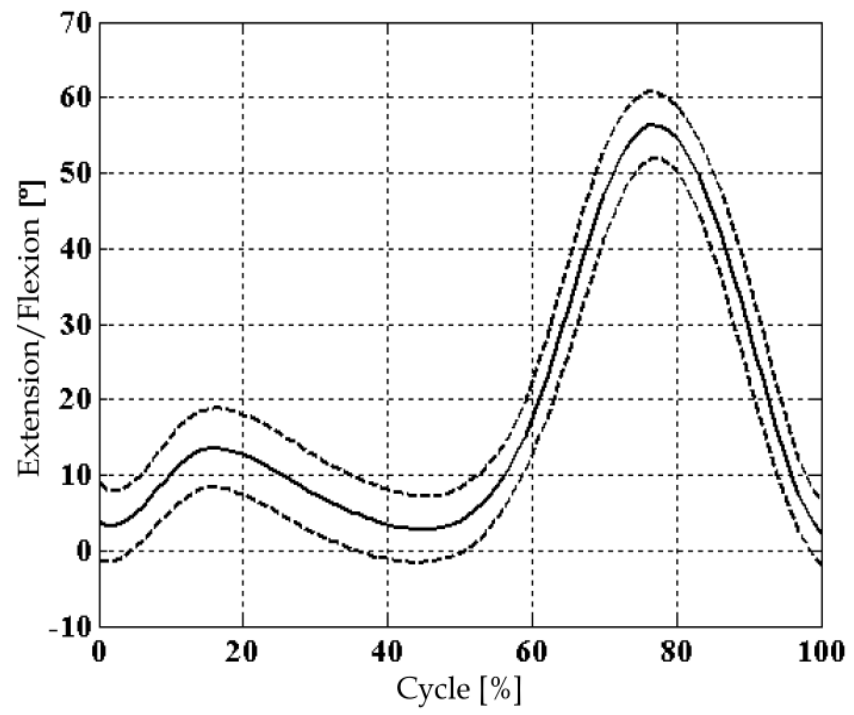

Fig. (7). Angular displacement of the knee in the sagittal plane (mean \pm deviation). 


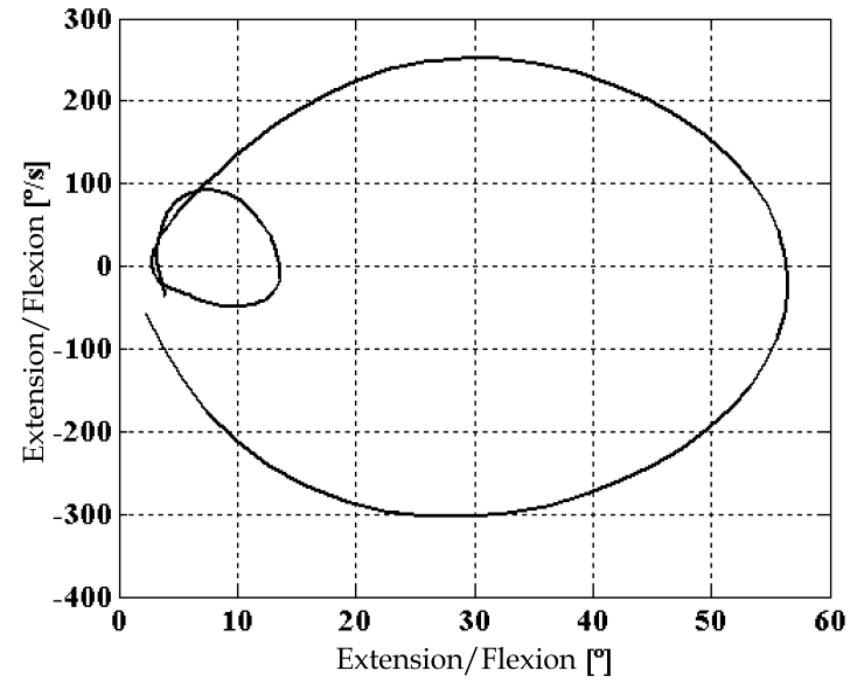

Fig. (8). Phase space of the knee in the sagittal plane.

In order to perform all determinants of gait, a 3D model is needed. In this work, to simplify the investigation, a 2D model that performs motions parallel only to the sagittal plane was considered (Fig. 9). This model characterises the three most important determinants of gait: the compass gait (1), the knee flexion of the stance leg (4), and the plantar flexion of the stance ankle (5).
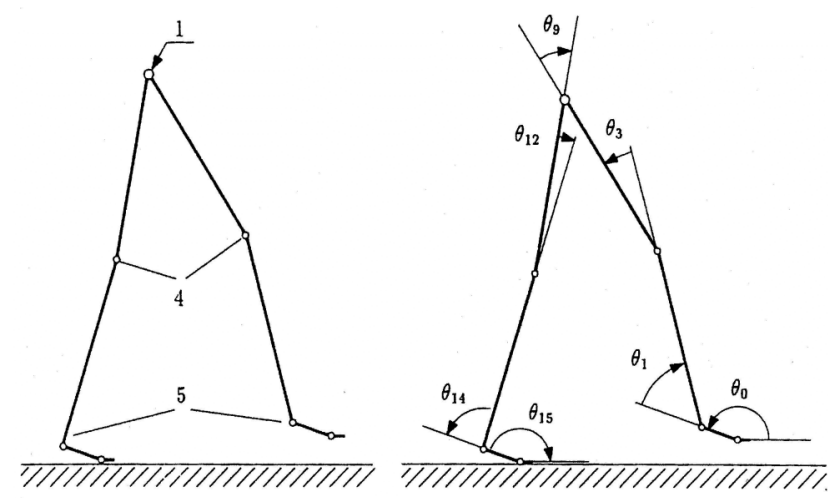

Fig. (9). Biped locomotor model with the most important determinants of gait and relative angles.

\section{NONLINEAR OSCILLATORS SYSTEM}

Oscillators are used to describe mechanisms that repeat its action periodically, such as: some neurons, electric circuits, waves, cells, etc. The behavior of an oscillator can be described by a differential equation, whose solution presents cyclical behavior. Thus, nonlinear oscillators can be represented by nonlinear differential equations.

One of the oscillators more known and used in diverse works about locomotion is the van der Pol oscillator. Another similar oscillator to the van der Pol is the Rayleigh oscillator, which is used in the oscillators system proposed in this work. The equation of Rayleigh used in the analyses was:

$\ddot{x}-\delta\left(1-q \dot{x}^{2}\right) \dot{x}+\Omega^{2}\left(x-x_{0}\right)=0 \quad \delta, q \geq 0$

where: $\delta, q$ and $\Omega$ correspond to the parameters of the oscillator.
Considering $y=\dot{x}$, we have the following autonomous system:

$\left\{\begin{array}{l}\dot{x}=y \\ \dot{y}=\delta\left(1-q y^{2}\right) y-\Omega^{2}\left(x-x_{0}\right)\end{array}\right.$

Choosing values for $\delta, q, \Omega$ and $x_{0}$, by means of a program that integrates the ordinary differential equations (ODE), is possible to plot the graphs of $x$ and $\dot{x}$ as function of the time and the trajectory in the phase space.

Taking into consideration oscillators with the same frequency, from the Eq. (1), considering a net of $n$-coupled Rayleigh oscillators, and adding a coupling term that relates the velocities of the oscillators, we have:

$$
\begin{aligned}
& \ddot{\theta}_{i}-\delta_{i}\left(1-q_{i} \dot{\theta}_{i}^{2}\right) \dot{\theta}_{i}+\Omega_{i}^{2}\left(\theta_{i}-\theta_{i o}\right)-\sum_{j=1}^{n} c_{i j}\left(\dot{\theta}_{i}-\dot{\theta}_{j}\right)=0 \\
& i=1,2, \ldots, n
\end{aligned}
$$

where: $\theta_{i}$ is the angle, and $\delta_{i}, q_{i}, \Omega_{i}$ and $c_{i, j}$ are the parameters of the system.

For small values of parameters determining the model nonlinearity, we will assume that the response is approximated by low frequency components from full range of harmonic response. Therefore periodic solutions can be expected, which can be approximated by:

$\theta_{i}=\theta_{i o}+A_{i} \cos \left(\omega t+\alpha_{i}\right)$

In this case, all oscillators have the same frequency $\omega$. Deriving the Eq. (4) and inserting the solutions in Eq. (3), by the method of harmonic balance [18], the following system of nonlinear algebraic equations are obtained:

$$
\left\{\begin{array}{l}
A_{i}\left(\Omega_{i}^{2}-\omega^{2}\right) \cos \alpha_{i}+A_{i} \delta_{i} \omega\left(1-\frac{3 \omega^{2} A_{i}^{2} q_{i}}{4}\right) \sin \alpha_{i}+ \\
\omega \sum_{j=1}^{n} c_{i, j}\left(A_{i} \sin \alpha_{i}-A_{j} \sin \alpha_{j}\right)=0 \\
A_{i}\left(\omega^{2}-\Omega_{i}^{2}\right) \sin \alpha_{i}+A_{i} \delta_{i} \omega\left(1-\frac{3 \omega^{2} A_{i}^{2} q_{i}}{4}\right) \cos \alpha_{i}+ \\
\omega \sum_{j=1}^{n} c_{i, j}\left(A_{i} \cos \alpha_{i}-A_{j} \cos \alpha_{j}\right)=0
\end{array}\right.
$$

With this system of equations, the parameters $q_{i}$ and $\Omega_{i}$ can be calculated:

$$
\begin{aligned}
& q_{i}=\frac{4}{3 \omega^{2} A_{i}^{2}}+\frac{4}{3 \omega^{2} A_{i}^{3} \delta_{i}} \sum_{j=1}^{n} c_{i, j}\left[A_{i}-A_{j} \cos \left(\alpha_{i}-\alpha_{j}\right)\right] \\
& i=1,2, \ldots, n \\
& \Omega_{i}=\sqrt{\omega^{2}-\frac{\omega}{A_{i}} \sum_{j=1}^{n} A_{j} c_{i, j} \sin \left(\alpha_{i}-\alpha_{j}\right)} \\
& i=1,2, \ldots, n
\end{aligned}
$$

Given the amplitude $A_{i}$ and $A_{j}$, phase $\alpha_{i}$ and $\alpha_{j}$, the frequency $\omega$, and the chosen values of $\delta_{i}$ and $c_{i, j}$, the value of the parameters $q_{i}$ and $\Omega_{i}$ can be calculated.

A similar procedure can be performed to the oscillators with integer relation of frequency. Oscillators of a coupling 
system, with frequency $\omega$, can be synchronised with other oscillators with frequency $n \omega$, where $n$ is an integer. Therefore, a net of coupled Rayleigh oscillators can be described as:

$$
\begin{aligned}
& \ddot{\theta}_{h}-\delta_{h}\left(1-q_{h} \dot{\theta}_{h}^{2}\right) \dot{\theta}_{h}+\Omega_{h}^{2}\left(\theta_{h}-\theta_{h o}\right)- \\
& \sum_{i=1}^{m} c_{h, i}\left[\dot{\theta}_{i}\left(\theta_{i}-\theta_{i o}\right)\right]-\sum_{k=1}^{n} c_{h, k}\left(\dot{\theta}_{h}-\dot{\theta}_{k}\right)=0
\end{aligned}
$$

where the term $c_{h, i}\left[\dot{\theta}_{i}\left(\theta_{i}-\theta_{i o}\right)\right]$ is responsible for the coupling between two oscillators with different frequencies, while the other term $c_{h, k}\left(\dot{\theta}_{h}-\dot{\theta}_{k}\right)$ makes the coupling between two oscillators with the same frequencies.

If the model nonlinearity is determined for small values of parameters, periodic solutions can be expected which can be approximated by the harmonic functions:

$\theta_{h}=\theta_{h o}+A_{h} \cos \left(2 \omega t+\alpha_{h}\right)$

$\theta_{i}=\theta_{i o}+A_{i} \cos \left(\omega t+\alpha_{i}\right)$

$\theta_{k}=\theta_{k o}+A_{k} \cos \left(2 \omega t+\alpha_{k}\right)$

Deriving the Eq. (9-11) and inserting the solutions in Eq. (8), by the method of harmonic balance [18], we have a system of nonlinear algebraic equations, which provide the parameters $q_{k}$ and $\Omega_{k}$ :

$$
\begin{gathered}
q_{h}=\frac{1}{3 \omega^{2} A_{h}^{2}}+\frac{1}{12 \omega^{2} A_{h}^{3} \delta_{h}} \sum_{i=1}^{m} A_{i}^{2} c_{h, i} \cos \left(\alpha_{h}-2 \alpha_{i}\right)+ \\
\frac{1}{3 \omega^{2} A_{h}^{3} \delta_{h}} \sum_{k=1}^{n} c_{h, k}\left[A_{h}-A_{k} \cos \left(\alpha_{h}-\alpha_{k}\right)\right] \\
\Omega_{h}=\sqrt{4 \omega^{2}+\frac{\omega}{2 A_{h}} \sum_{i=1}^{m} A_{i}^{2} c_{h, i} \sin \left(\alpha_{h}-2 \alpha_{i}\right)-} \\
\frac{2 \omega}{A_{h}} \sum_{k=1}^{n} A_{k} c_{h, k} \sin \left(\alpha_{h}-\alpha_{k}\right)
\end{gathered}
$$

Given the amplitude $A_{h}, A_{i}$ and $A_{k}$, phase $\alpha_{h}, \alpha_{i}$ and $\alpha_{k}$, the frequency $\omega$, and the chosen values of $\delta_{h}, c_{h, i}$ and $c_{h, k}$, the value of the parameters $q_{h}$ and $\Omega_{h}$ can be calculated.

\section{ANALYSIS, RESULTS AND DISCUSSION}

To study the behavior of knee angles $\theta_{3}$ and $\theta_{12}$, and hip angle $\theta_{9}$ (Fig. 9), a set of three coupled oscillators had been used. These oscillators are mutually coupled by terms that determine the influence of each oscillator on the others.

Applying the Eq. (8) in this configuration, the system can be described by the equations:

$$
\begin{aligned}
& \ddot{\theta}_{3}-\delta_{3}\left(1-q_{3} \dot{\theta}_{3}^{2}\right) \dot{\theta}_{3}+\Omega_{3}^{2}\left(\theta_{3}-\theta_{3 o}\right)- \\
& c_{3,9}\left[\dot{\theta}_{9}\left(\theta_{9}-\theta_{9 o}\right)\right]-c_{3,12}\left(\dot{\theta}_{3}-\dot{\theta}_{12}\right)=0 \\
& \ddot{\theta}_{9}-\delta_{9}\left(1-q_{9} \dot{\theta}_{9}^{2}\right) \dot{\theta}_{9}+\Omega_{9}^{2}\left(\theta_{9}-\theta_{9 o}\right)- \\
& c_{9,3}\left[\dot{\theta}_{3}\left(\theta_{3}-\theta_{3 o}\right)\right]-c_{9,12}\left[\dot{\theta}_{12}\left(\theta_{12}-\theta_{12 o}\right)\right]=0 \\
& \ddot{\theta}_{12}-\delta_{12}\left(1-q_{12} \dot{\theta}_{12}^{2}\right) \dot{\theta}_{12}+\Omega_{12}^{2}\left(\theta_{12}-\theta_{12 o}\right)- \\
& c_{12,9}\left[\dot{\theta}_{9}\left(\theta_{9}-\theta_{9 o}\right)\right]-c_{12,3}\left(\dot{\theta}_{12}-\dot{\theta}_{3}\right)=0
\end{aligned}
$$

The synchronised harmonic functions, corresponding to the desired movements, can be writing as:

$\theta_{3}=\theta_{3 o}+A_{3} \cos \left(2 \omega t+\alpha_{3}\right)$

$\theta_{9}=A_{9} \cos \left(\omega t+\alpha_{9}\right)$

$\theta_{12}=\theta_{12 o}+A_{12} \cos \left(2 \omega t+\alpha_{12}\right)$

Considering $\alpha_{3}=\alpha_{9}=\alpha_{12}=0$ and deriving the Eq. (1719), inserting the solution into the differential Eq. (14-16), the necessary parameters of the oscillators $\left(q_{i}\right.$ and $\Omega_{i}, i \in\{3$, $9,12\})$ can be determined. Then:

$$
q_{3}=\frac{4 c_{3,12}\left(A_{3}-A_{12}\right)+4 A_{3} \delta_{3}+A_{9}^{2} c_{3,9}}{12 \omega^{2} A_{3}^{3} \delta_{3}}
$$

$\Omega_{3}=2 \omega$

$q_{9}=\frac{4}{3 \omega^{2} A_{9}^{2}}$

$\Omega_{9}=\omega$

$q_{12}=\frac{4 c_{12,3}\left(A_{12}-A_{3}\right)+4 A_{12} \delta_{12}+A_{9}^{2} c_{12,9}}{12 \omega^{2} A_{12}^{3} \delta_{12}}$

$\Omega_{12}=2 \omega$

From equations (14-16) and (20-25), and using the MAT$\mathrm{LAB}$, the graphs shown in Figs. (10) and (11) were generated, and present, respectively, the behavior of the angles as function of the time and the stable limit cycles of the oscillators.

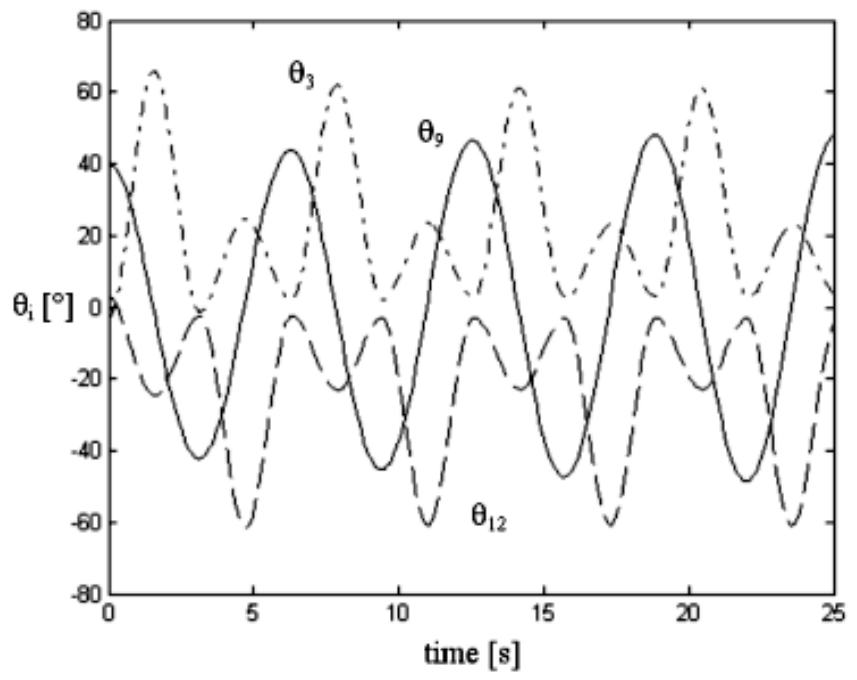

Fig. (10). Behaviour of $\theta_{3}, \theta_{9}$ and $\theta_{12}$ as function of the time.

These results were obtained by using the parameters showed in Table $\mathbf{1}$, as well as the initial values provided by Table 2. All values were experimentally determined.

Comparing Figs. (10) and (11) with the experimental results presented in Figs. (5-8), despite of the different amplitudes demonstrated by the experimental tests and by nonlinear oscillators system, taking into consideration only the cycle of locomotion, where $100 \%$ of cycle in the Figs. (5) and (7) is equivalent to $6.28 \mathrm{~s}$ in Fig. (10), the graph gener- 
ated by oscillators system represent the pattern of behavior of the elements (hip and knees), being able to be used to generate the approximate trajectories for the legs. This confirms the possibility of the use of coupled Rayleigh oscillators in the modeling of the central pattern generator.

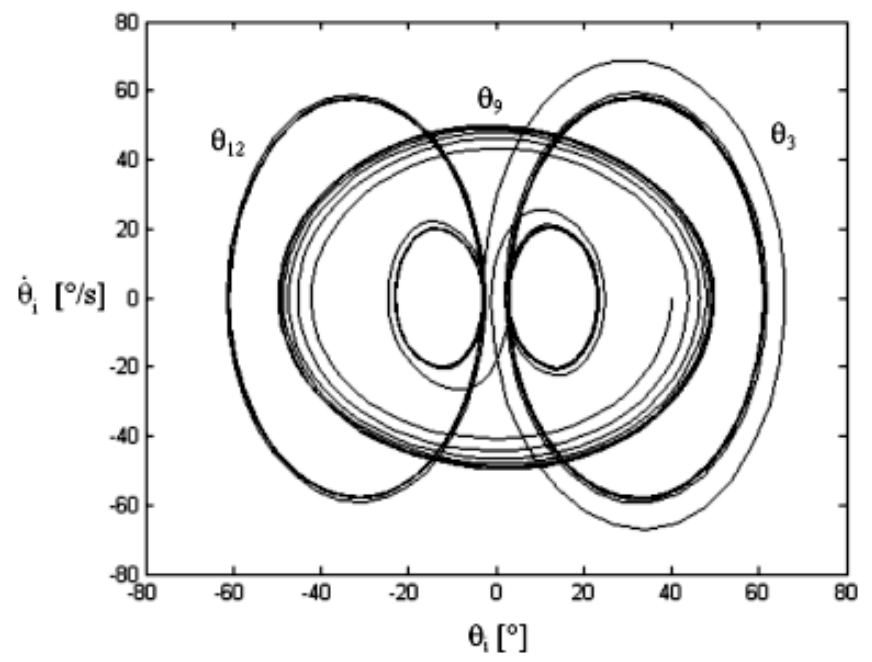

Fig. (11). Trajectories in the phase space (stable limit cycles).

Table 1. Parameters of Rayleigh Oscillators

\begin{tabular}{|c|c|c|c|c|c|c|c|c|}
\hline$c_{3,9}$ & $c_{9,3}$ & $c_{3,12}$ & $c_{12,3}$ & $c_{9,12}$ & $c_{12,9}$ & $\varepsilon_{3}$ & $\varepsilon_{9}$ & $\varepsilon_{12}$ \\
\hline \hline 0.001 & 0.001 & 0.1 & 0.1 & 0.001 & 0.001 & 0.01 & 0.1 & 0.01 \\
\hline
\end{tabular}

Table 2. Experimental Initial Values

\begin{tabular}{|c||c|c|c|c|c|c|}
\hline Cycle & $\boldsymbol{A}_{\mathbf{3}}$ & $\boldsymbol{A}_{\mathbf{9}}$ & $\boldsymbol{A}_{\mathbf{1 2}}$ & $\boldsymbol{\theta}_{\mathbf{3 0}}$ & $\boldsymbol{\theta}_{\mathbf{9} \mathbf{0}}$ & $\boldsymbol{\theta}_{\mathbf{1 2 0}}$ \\
\hline \hline $0<\omega t \leq \pi$ & -29 & 50 & 10 & 32 & 0 & -13 \\
\hline$\pi<\omega t \leq 2 \pi$ & -10 & 50 & 29 & 13 & 0 & -32 \\
\hline
\end{tabular}

Before implementing the oscillators system, from the biped locomotor model and applying the system to generate the degrees of freedom of the biped, a simulation was performed take into consideration the Eq. (14-16) and Eq. (2025), with the values showing in Tables 1-3. The simulations presented here were performed only through a kinematical analysis. Fig. (12) presents the biped gait for angular amplitude of the hip equal to $50^{\circ}$.

Table 3. Model Dimensions

\begin{tabular}{|c|c|}
\hline Model Part & Length [m] \\
\hline \hline Foot & 0.14 \\
\hline Tibia & 0.37 \\
\hline Femur & 0.37 \\
\hline
\end{tabular}

In this case, the adopted value for amplitude of the hip angle provides a step length equal to $0.63 \mathrm{~m}$. A alteration of this amplitude value cause a change of step length, conse- quently modifying the gait. Thus, the pattern generator system makes possible the change of step length by means of amplitude alteration.

Fig. (13) presents the biped gait for angular amplitude of the hip equal to $40^{\circ}$. In this case, the step length is equal to $0.5 \mathrm{~m}$. Fig. (14) presents the biped gait for angular amplitude of the hip equal to $30^{\circ}$. In this case, the step length is equal to $0.38 \mathrm{~m}$.

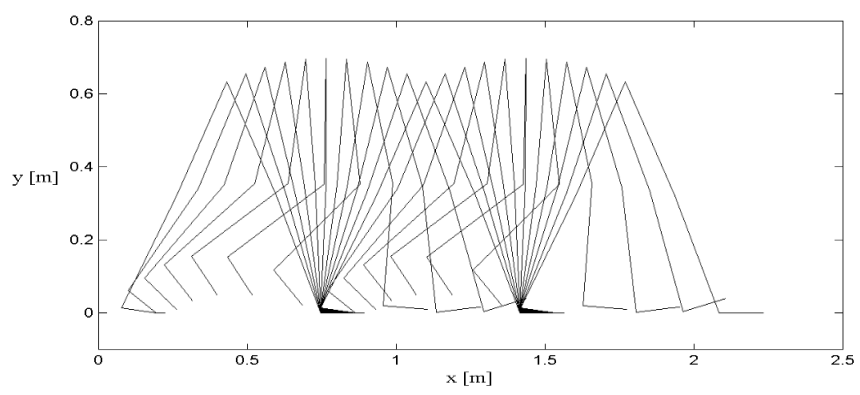

Fig. (12). Gait for $A_{h}=50^{\circ}$.

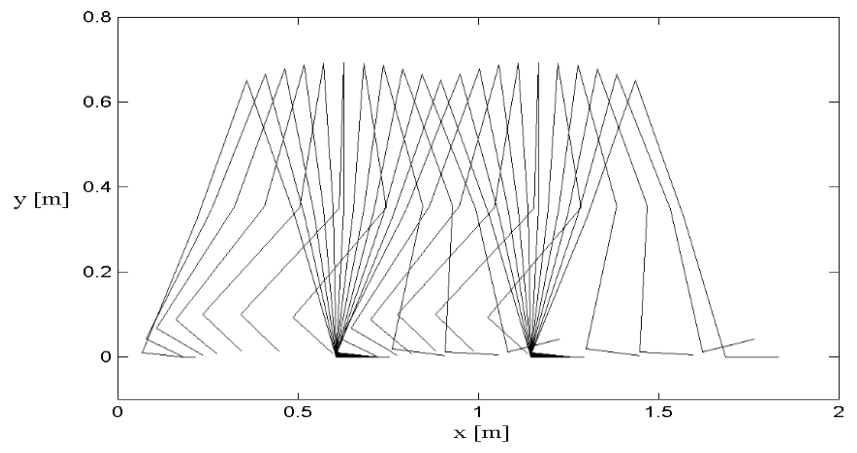

Fig. (13). Gait for $A_{h}=40^{\circ}$.

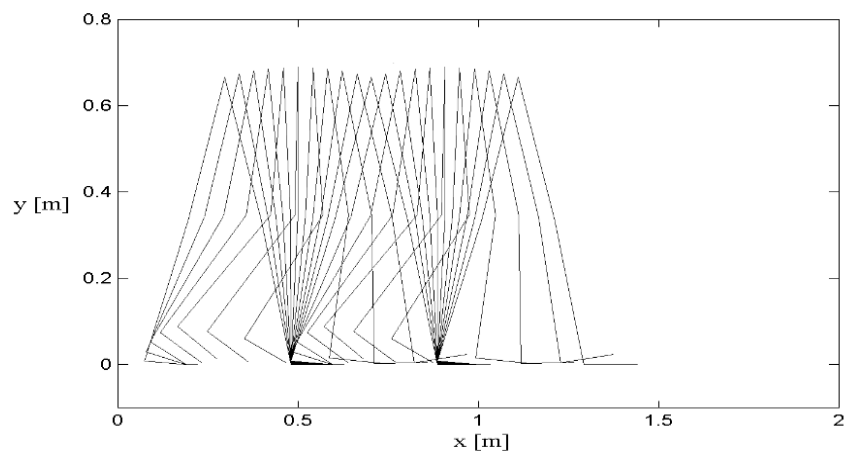

Fig. (14). Gait for $A_{h}=30^{\circ}$.

Besides the change of step length, the oscillators system makes also possible the change in the gait frequency, which can be modify by means of the Eq. (17-19), choosing a new value for $\omega$. Then, modifying the value $\omega$ from 1 to 2 , we have logically a duplicated gait frequency (Figs. 15 and 16).

\section{CONCLUSION}

From presented results and their analysis and discussion, we come to the following conclusions about the simulation of the biped gait by means of coupled oscillators: 1) The use of mutually coupled Rayleigh oscillators can represent an excellent way to signal generation, allowing their application for feedback control of a walking machine by synchronisa- 
tion and coordination of the lower extremities. 2) The biped locomotor model is able to characterise three of the six most important determinants of human gait. 3) Modification of the step length and gait frequency can be obtained by means of the change of few parameters in the oscillators. The gait frequency can be modified using the Eq. (17-19), by choosing a new value for $\omega$. The step length can be modified by changing the angles $\theta_{9}$ and $\theta_{12}$, being the parameters $q_{i}$ and $\Omega_{i}, i \in$ $\{3,9,12\}$, responsible for the gait transitions.

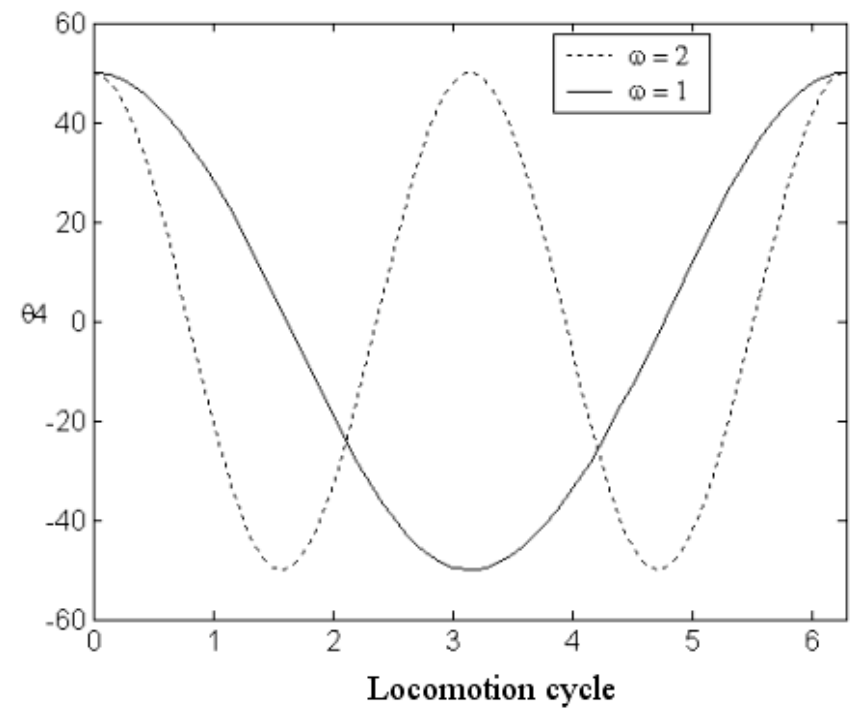

Fig. (15). Hip behavior with modification of the gait frequency.

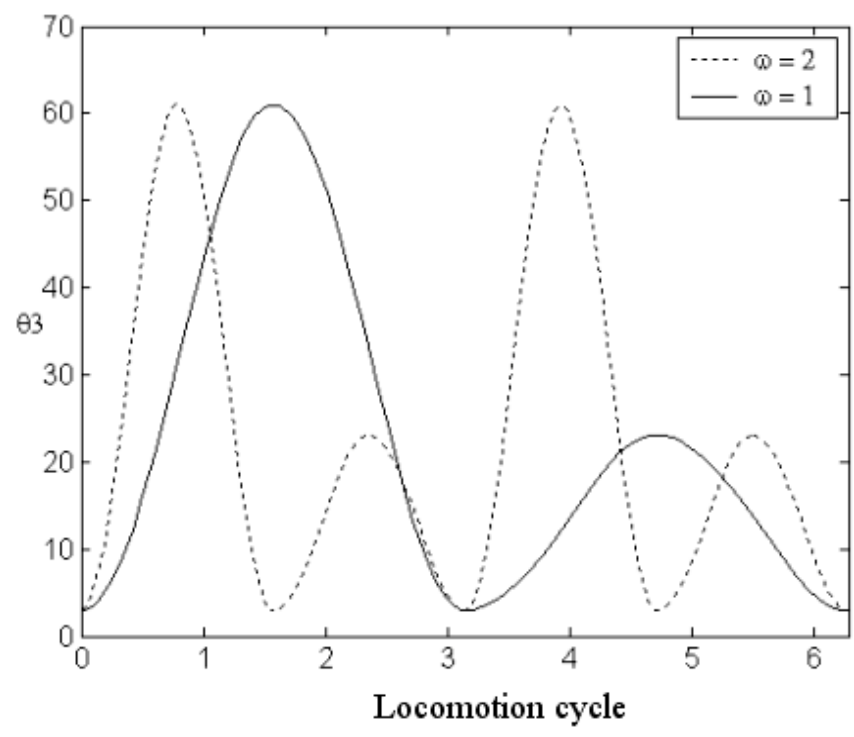

Fig. (16). Knee behavior with modification of the gait frequency.

In future works, we intend to study the behavior of the ankles, as well as simulate the behavior of the hip and knees in other anatomical planes, thus increasing the network of coupled oscillators, looking for to characterise all determi- nants of gait, and consequently simulate with more details the biped gait.

\section{ACKNOWLEDGMENTS}

The author would like to express your gratitude to FAPERJ (Fundação Carlos Chagas Filho de Amparo à Pesquisa do Estado do Rio de Janeiro), governmental entity promoter of the scientific and technological development, for the financial support for purchase of the equipment used in the course of this present research.

\section{REFERENCES}

[1] M. M. Lyons, "Central Pattern Generation of Locomotion: A Review of the Evidence", Phys. Ther., vol. 82 no. 1, 2002.

[2] B. Calancie, B. N. Shropshire, P. Jacobs, K. Willer, G. Zych, and B. A. Green, "Involuntary stepping after chronic spinal cord injury. Evidence for a central rhythm generator for locomotion in man", Brain, vol. 117(Pt 5), pp. 1143-1159, 1994.

[3] M. R. Dimitrijevic, Y. Gerasimenko, and M. M. Pinter, "Evidence for a spinal central pattern generator in humans", Ann. NY Acad. Sci., vol. 860, pp. 360-376, 1998.

[4] M. M. Pinter, and M. R. Dimitrijevic, "Gait after spinal cord injury and the central pattern generator for locomotion", Spinal Cord, vol 37, no. 8, pp. 531-537, 1999.

[5] J. S. Bay, and H. Hemami, "Modelling of a neural pattern generator with coupled nonlinear oscillators", IEEE Trans. Biomed. Eng., vol. 34 , pp. 297-306, 1987.

[6] M. S. Dutra, Bewegungskoordination und Steuerung einer zweibeinigen Gehmaschine, Shaker Verlag, Germany, 1995.

[7] M. S. Dutra, A. C. de Pina Filho, and V. F. Romano, Modeling of a Bipedal Locomotor Using Coupled Nonlinear Oscillators of Van der Pol, Biol. Cybern., vol. 88, no. 4, pp. 286-292, 2003.

[8] Z. Yang, and F. M. G. França, "A generalized locomotion CPG architecture based on oscillatory building blocks", Biol. Cybern., vol. 89, pp. 34-42, 2003.

[9] A. C. de Pina Filho, Study of Mutually Coupled Nonlinear Oscillators Applied in the Locomotion of a Bipedal Robot, D.Sc. Thesis, PEM/COPPE/UFRJ, Brazil, 2005.

[10] L. Righetti, and A. J. Ijspeert, "Programmable central pattern generators: an application to biped locomotion control", In: IEEE International Conference on Robotics and Automation, Orlando, Florida, pp. 1585-1590, 2006.

[11] A. Heralic, K. Wolff, and M. Wahde, "Central Pattern Generators for Gait Generation in Bipedal Robots, In: Humanoid Robots - New Developments, Edited by A. C. de Pina Filho, I-Tech Education and Publishing, Viena, Austria, pp. 285-304, 2007.

[12] A. Sproewitz, R. Moeckel, J. Maye, and A. J. Ijspeert, "Learning to Move in Modular Robots using Central Pattern Generators and Online Optimization", Int. J. Robot. Res., vol. 27, no. 3-4, pp. 423443, 2008.

[13] M. L. Brandão, As Bases Biológicas do Comportamento: Introdução à Neurociência, Editora Pedagógica Universitária (EPU), 225 p., Brazil, 2004.

[14] V. Dietz, "Spinal cord pattern generators for locomotion", Clin. Neurophysiol., vol. 114, pp. 1379-1389, 2003.

[15] I. A. Kapandji, Fisiologia articular: esquemas comentados de mecânica humana, Ed. 4, vol. 2, Editora Manole, Brazil, 1980.

[16] L. S. C. Raptopoulos, Estudo e Desenvolvimento de Equipamento de Baixo Custo para Análise da Marcha de Amputados, D.Sc. Thesis, PEM/COPPE/UFRJ, Brazil, 2003.

[17] J. B. Saunders, V. Inman, and H. D. Eberhart, "The Major Determinants in Normal and Pathological Gait", J. Bone Joint Surg., vol. 35A, 1953.

[18] A. H. Nayfeh, and D. T. Mook, Nonlinear oscillations, John Wiley \& Sons, Inc., 1979. 